\title{
Síndrome X frágil: presentación clínica, patología y tratamiento
}

María Jimena Salcedo-Arellano, Randi J. Hagerman y Verónica Martínez-Cerdeño*

Universidad de California en Davis, Escuela de Medicina, Instituto de Investigación Médica de los Trastornos del Neurodesarrollo. Sacramento, California, Estados Unidos

\section{Resumen}

El síndrome $X$ frágil es la condición monogenética que produce más casos de autismo y de discapacidad intelectual. La repetición de tripletes CGG (> 200) y su metilación conllevan el silenciamiento del gen FMR1. La proteína FMRP (producto del gen FMR1) interacciona con los ribosomas, controlando la traducción de mensajeros específicos y su pérdida produce alteraciones de la conectividad sináptica. El tamizaje de síndrome $X$ frágil se realiza por reacción en cadena de la polimerasa. La recomendación actual de la Academia Americana de Pediatría es realizar pruebas a quienes presenten discapacidad intelectual, retraso global del desarrollo o antecedentes familiares de afección por la mutación o premutación. Países hispanos como Colombia, Chile y España reportan altas prevalencias de síndrome $X$ frágil y han creado asociaciones o corporaciones nacionales de $X$ frágil que buscan acercar a los pacientes a redes disponibles de diagnóstico y tratamiento.

PALABRAS CLAVE: Síndrome X frágil. Gen FMR1. Proteína FMRP.

\section{Fragile $X$ syndrome: clinical presentation, pathology and treatment}

\begin{abstract}
Fragile $X$ syndrome is the monogenetic condition that produces more cases of autism and intellectual disability. The repetition of CGG triplets (> 200) and their methylation entail the silencing of the FMR1 gene. The FMRP protein (product of the FMR1 gene) interacts with ribosomes by controlling the translation of specific messengers, and its loss causes alterations in synaptic connectivity. Screening for fragile $X$ syndrome is performed by polymerase chain reaction. Current recommendation of the American Academy of Pediatrics is to test individuals with intellectual disability, global developmental retardation or with a family history of presence of the mutation or premutation. Hispanic countries such as Colombia, Chile and Spain report high prevalence of fragile $X$ syndrome and have created fragile $X$ national associations or corporations that seek to bring patients closer to available diagnostic and treatment networks.
\end{abstract}

KEY WORDS: Fragile X syndrome. FMR1 gene. FMRP protein.

\section{Introducción}

El síndrome $X$ frágil (SXF) es una condición de repetición de nucleótidos no mendeliana. EI SXF se debe a la pérdida de función del gen FMR1 (fragile $x$ mental retardation 1). El gen FMR1 se encuentra en el cromosoma Xq27.3 y codifica la proteína FMRP, cuya función es controlar la traducción de mensajeros específicos. La repetición de tripletes CGG (> 200 repeticiones) y la metilación del promotor conllevan el silenciamiento del gen. Sin embargo, el mecanismo biológico responsable de la presentación del SXF no se conoce del todo. Aproximadamente, $30 \%$ de las niñas y $90 \%$ de los niños afectados con la mutación
Correspondencia:

*Verónica Martínez-Cerdeño

E-mail: vmartinezcerdeno@ucdavis.edu
Gac Med Mex. 2020;156:60-66

Disponible en PubMed

www.gacetamedicademexico.com

0016-3813/@ 2019 Academia Nacional de Medicina de México, A.C. Publicado por Permanyer. Éste es un artículo open access bajo la licencia CC BY-NC-ND (http://creativecommons.org/licenses/by-nc-nd/4.0/). 
completa presentan discapacidad intelectual y $60 \%$ de los niños son diagnosticados con trastornos del espectro autista (TEA). Los trastornos de ansiedad ocurren entre 70 y $80 \%$ de las personas con SXF.

La prevalencia más aceptada del SXF es de aproximadamente uno en 5000 hombres y una en 4000 a 8000 mujeres. Sin embargo, aún no hay un consenso global debido a la complejidad del diagnóstico molecular y la variedad en la presentación clínica de quienes no están severamente afectados. ${ }^{1}$ Se han reportado prevalencias mucho más altas en España ${ }^{2}$ y Colombia, donde recientemente se reportó un conglomerado genético con la prevalencia más alta del síndrome hasta la fecha; ${ }^{3}$ así como una casi inexistente en China, donde se especula que la falta de investigación y especialización clínica en áreas del neurodesarrollo es la causa principal de la escasez diagnóstica del síndrome.

\section{Bases biológicas del síndrome X frágil}

El mecanismo biológico exacto responsable de la presentación del SXF no se conoce, sin embargo, se sabe que reside en la capacidad de la proteína FMRP de unión a ARN y a proteínas. Específicamente, FMRP se une a ribosomas y está presente en los compartimentos sinápticos, donde controla la traducción de mensajeros específicos. La pérdida de FMRP produce alteraciones de la conectividad sináptica en las neuronas, que se traducen en los síntomas específicos del SXF. Estas alteraciones de la conectividad sináptica se ponen de manifiesto en el cerebro con la disminución de la cantidad de dendritas y espinas en las neuronas de pacientes con SXF.

La falta de FMRP en neuronas conlleva una expresión exacerbada de receptores de glutamato, tanto metabotrópicos ( mGluR $_{5}$ ) como ionotrópicos (AMPA y NMDA). ${ }^{4}$ Las proteínas de síntesis, degradación y trasporte del ácido $\gamma$-aminobutírico (GABA) y los receptores de GABA también están reducidas. ${ }^{5}$ No se sabe exactamente cómo estos cambios en los sistemas de neurotransmisores afectan la morfología de dendritas y espinas en las neuronas, pero se sospecha que están relacionadas. El papel de la proteína FMRP en las células gliales es menos conocido, pero se sabe que en SXF la proteína FMRP regula la traducción de mGluR5 en astrocitos ${ }^{6}$ y la producción de mielina en oligodendrocitos. ${ }^{7}$ Durante el desarrollo prenatal, las células de la glía radial contienen FMRP, que interviene en el transporte activo de ARN mensajero a lo largo de la fibra glial. $^{8}$ Un cambio en cualquiera de estos mecanismos puede colaborar al desarrollo de las alteraciones cognitivas en los pacientes con SXF.

FMRP ha sido relacionada con la regulación de canales iónicos. FMRP se une al C-terminal de los canales Slack, activados por potasio. La activación de los canales Slack contribuye a los patrones de activación de una gran variedad de neuronas, lo que sugiere que las alteraciones observadas en el SXF pueden ser generadas por patrones de actividad alterados. $^{9} \mathrm{~A}$ su vez, FMRP también puede regular la liberación de neurotransmisores a través de la modulación del potencial de acción vía canales de potasio de larga conducción activados por calcio (canales BK). ${ }^{10}$

La presencia de una pequeña fracción de FMRP en el núcleo celular indica que dicha proteína puede tener funciones previamente no reconocidas. De hecho, varios estudios han desvelado funciones relacionadas con la expresión del ADN y la función genómica, como en la estabilización del ADN, la regulación epigenética del ADN, la regulación del ARN nuclear y la respuesta al daño del ADN..$^{11}$

La proteína precursora del $\beta$ amiloide (APP) también ha sido relacionada con el SXF, a través de un mecanismo dependiente del receptor $m$ GluR $_{5}$. APP es procesada por secretasas que producen $\beta$ amiloide $(A \beta)$, péptido predominante en las placas seniles en la enfermedad de Alzheimer. ${ }^{12}$

\section{Presentación clínica}

Los afectados con la mutación completa del gen FMR1 presentan características fenotípicas especiales que incluyen cara alargada, orejas grandes y prominentes, hipermovilidad articular y macroorquidismo. ${ }^{13}$ Más de $90 \%$ de los niños afectados presentan retraso del desarrollo y aproximadamente el 50-60\% son diagnosticados con TEA..$^{14}$ Durante el transcurso de su vida, tanto hombres como mujeres presentan alteraciones del comportamiento comúnmente asociadas con el síndrome, usualmente de inicio durante la infancia: la ansiedad y el trastorno de atención e hiperactividad (TDAH) son las más prevalentes; aunque los desórdenes compulsivos como la hiperfagia y la agresividad también son comunes (Tabla 1). Además de las alteraciones comportamentales y los problemas de aprendizaje y adaptación social, de 15 a $20 \%$ de los pacientes con SXF presentan convulsiones, más prevalentes en aquellos con autismo; más de $30 \%$ tiene problemas de obesidad, alteraciones del sueño y alguna disfunción gastrointestinal, incluyendo reflujo gastroesofágico. El 
Tabla 1. Características clínicas

\begin{tabular}{|c|c|c|}
\hline & Características clínicas & Prevalencia \\
\hline Físicas & $\begin{array}{l}\text { Cara alargada } \\
\text { Macrocefalia } \\
\text { Orejas prominentes } \\
\text { Mandíbula prominente } \\
\text { Pies planos } \\
\text { Macroorquidismo } \\
\text { Hipermovilidad articular }\end{array}$ & $\begin{array}{c}83 \% \text { más común en adultos } \\
50-81 \% \\
75 \% \\
80 \% \text { en adultos } \\
29-69 \% \\
95 \% \text { desde la adolescencia } \\
50-70 \% \text { más común en niños }\end{array}$ \\
\hline Psicológicas/psiquiátricas & $\begin{array}{l}\text { TDAH } \\
\text { TEA } \\
\text { Ansiedad } \\
\text { Agresividad }\end{array}$ & $\begin{array}{c}80 \% \text { niños y } 40 \% \text { niñas } \\
50-60 \% \text { niños y } 20 \% \text { niñas } \\
58-86 \% \\
40 \% \text { niños y } 10-15 \% \text { niñas }\end{array}$ \\
\hline Desarrollo & $\begin{array}{l}\text { Discapacidad intelectual } \\
\text { Déficit del lenguaje }\end{array}$ & $\begin{array}{l}85 \% \text { niños y } 25-30 \% \text { niñas } \\
100 \% \text { niños y } 60-75 \% \text { niñas }\end{array}$ \\
\hline Otros & $\begin{array}{l}\text { Estrabismo } \\
\text { Otitis recurrente } \\
\text { Problemas gastrointestinales } \\
\text { Obesidad } \\
\text { Convulsiones }\end{array}$ & $\begin{array}{c}\text { 8-30\% } \\
50-75 \% \text { en la infancia } \\
30 \% \\
30-60 \% \\
15-20 \%\end{array}$ \\
\hline
\end{tabular}

Adaptado de referencia 14. TDAH = trastorno de atención e hiperactividad, TEA = trastornos del espectro autista.

estrabismo y la otitis media recurrente son problemas comunes durante la primera infancia.

El fenotipo tiene algunas variantes. Los más afectados con la mutación son los hombres; las mujeres presentan un fenotipo atenuado por el índice de activación del segundo cromosoma $\mathrm{X}$ no afectado. Más de $70 \%$ de las mujeres tiene un coeficiente intelectual bajo, si bien considerado promedio comparado con la población general y en menor proporción comparado con los hombres que presentan problemas de lenguaje..$^{13}$ La segunda variante son los mosaicos, que presentan algunas líneas celulares con la mutación completa y otras en el rango de premutación, lo que los expone al riesgo de padecer los problemas propios de la premutación como el síndrome de temblor/ataxia (FXTAS):;15,16 o algunas líneas celulares con metilación $y$, por ende, el gen silenciado y otras sin metilación, en este caso los afectados también presentan menor grado de afectación cognitiva. ${ }^{17}$

Además de las características fenotípicas comúnmente reconocidas, los afectados presentan anomalías del tejido conectivo, atribuidas a que FMRP regula componentes esenciales de la matriz extracelular. Además de las alteraciones musculoesqueléticas más comunes, como la hiperextensión de las articulaciones metacarpofalángicas, los pies planos y escoliosis, se han descrito alteraciones en los sistemas cardiovascular y genitourinario. ${ }^{18}$

Imágenes de resonancia magnética del cerebro de los pacientes con SXF demuestran que por lo general el cerebro es más grande de lo normal y con incremento en el tamaño de los ventrículos laterales. El vermis cerebelar presenta hipoplasia, una de las características más representativa, que puede ir acompañada de la reducción del cerebelo entero y alteraciones de los pedúnculos cerebelosos. Además, el núcleo caudado, sobre todo la cabeza, es más grande, principalmente en hombres. El hipocampo también está agrandado en pacientes jóvenes. Por el contrario, la ínsula y la amígdala son más pequeñas. Asimismo, el fascículo uncinado también presenta alteraciones de la materia blanca. ${ }^{13}$

\section{Interacción entre SXF, autismo y trastorno de déficit de atención e hiperactividad}

Existe una relación estrecha entre la presencia de SXF, TEA y TDAH. Aproximadamente $2 \%$ de todos los casos diagnosticados de trastornos del espectro autista (TEA) son atribuibles al SXF; mientras que más de $60 \%$ de los niños con SXF son diagnosticados con TDAH, TEA o los dos. El SXF es la principal causa genética conocida de los TEA, sin embargo, solo $20 \%$ de los casos de autismo son reconocidos como el resultado de mutaciones monogénicas y solo 2 a $6 \%$ se debe a la mutación del gen FMR1. Los afectados por las dos morbilidades, como sucede en 50 a $60 \%$ de los niños y $20 \%$ de las niñas con SXF, presentan un compromiso más severo tanto del déficit cognitivo y del lenguaje como en los problemas del comportamiento. ${ }^{19}$ Los ensayos clínicos controlados han demostrado que a pesar de que SXF y TEA comparten 


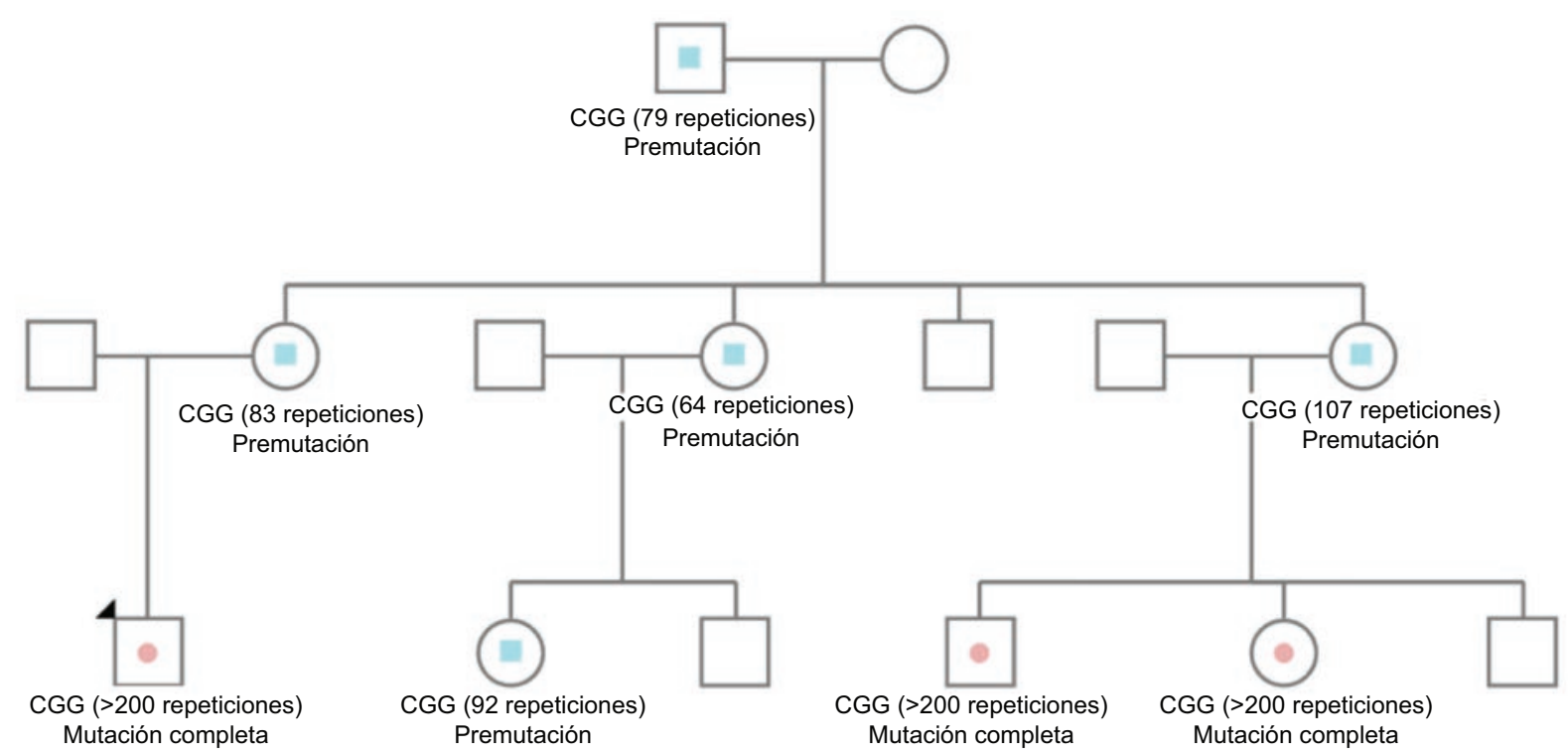

Figura 1. Árbol familiar. Al hacer el diagnóstico molecular se recomienda realizar diagnóstico en cascada a todos los miembros de la familia inmediata. El hombre portador de la premutación en la primera generación pasa la premutación a $100 \%$ de sus hijas mujeres, mientras que sus hijos varones no serán portadores de la premutación o de la mutación completa. Los hijos e hijas de las mujeres en la segunda generación tienen $50 \%$ de probabilidad de tener la premutación o la mutación completa. Solo las mujeres con la premutación tienen la capacidad de expandir la mutación completa tanto a sus hijos varones como mujeres y estos de presentar el SXF en la tercera generación.

sintomatología psiquiátrica, los afectados no responden con la misma eficacia a tratamientos específi$\cos ,{ }^{20,21}$ por lo cual se sugiere que los mismos síntomas emergen de distintos mecanismos.

\section{Diagnóstico}

La edad aproximada del diagnóstico del SXF es de 36 meses, ${ }^{22}$ a pesar de que la mayoría de los padres reporta reconocer algún tipo de retraso en el neurodesarrollo durante el primer año de vida. El tamizaje de poblaciones de alto riesgo puede realizarse por prueba de reacción en cadena de la polimerasa (PCR), de relativo bajo costo que requiere una sola gota sanguínea. El método utiliza un cebador quimérico que apunta aleatoriamente dentro de la región ampliada de CGG en el gen FMR1. ${ }^{23}$ Este método se ha utilizado exitosamente en varios estudios poblacionales. ${ }^{2,3}$ La prueba confirmatoria del diagnóstico es Southern Blot. Tejada hizo la evaluación completa de las ventajas y controversias de la prevención del SXF haciendo uso del diagnóstico prenatal. ${ }^{24}$ En 2017, Riley y Wheeler describieron la problemática del establecimiento del tamizaje posnatal en Estados Unidos. ${ }^{25}$ La recomendación actual de la Academia Americana de Pediatría es realizar pruebas genéticas a los niños con discapacidad intelectual o retraso global del desarrollo. ${ }^{26}$ De encontrarse un caso nuevo de SXF debe realizarse diagnóstico en cascada a todos los miembros de la familia inmediata, con el fin de identificar a los portadores de la premutación que tienen el potencial de expandir la mutación completa a sus descendientes (Figura 1). Recientemente Lubala et al. realizaron un metaanálisis en el que se incluyeron 10 estudios de tamizaje y se propuso una puntuación clínica para las siete características más específicas del SXF; esta lista toma en consideración las diferencias, sobre todo faciales, que pueden encontrarse en diferentes grupos étnicos. Esta herramienta clínica es de suma importancia en áreas donde no todos los afectados con discapacidad intelectual o TEA pueden someterse a pruebas genéticas debido a limitación de los recursos (Tabla 2). ${ }^{27}$

\section{Diagnóstico en países hispanos}

A pesar de la recomendación de realizar pruebas genéticas en niños con discapacidad intelectual o retraso global del desarrollo y en aquellos cuyas familias están afectadas, estas pruebas no se realizan en numerosos países hispanos. Las pruebas genéticas diagnósticas están disponibles y varios países en Latinoamérica han reportado estudios de prevalencia del SXF, sin embargo, determinar la prevalencia real de los desórdenes genéticos es difícil debido a que en numerosas naciones hispanas no existe un registro oficial nacional. Países como Chile, Brasil, Colombia, Argentina, Perú y España han hecho visible la 
Tabla 2. Lista de verificación clínica para el síndrome X frágil

\begin{tabular}{|c|c|c|}
\hline Caracteristica & \multicolumn{2}{|c|}{ Puntuación } \\
\cline { 2 - 3 } & 1 & 2 \\
\hline
\end{tabular}

Piel suave y aterciopelada en las palmas de las $X$ manos con exceso de piel en el dorso de la mano

Pies planos $x$

Orejas grandes y prominentes $x$

Pliegue plantar $x$

Macroorquidismo* $X$

Historia familiar de discapacidad intelectual $X$

Comportamiento autista $x$

Total

46

*Varones después de la pubertad. La puntuación máxima es de 10 puntos para varones después de la pubertad y de nueve para varones antes de la pubertad o mujeres. En pacientes con puntuación superior a 5 debe considerarse el diagnóstico molecular de SXF. Adaptado de referencia 27.

necesidad de implementar mejores procesos de tamizaje y diagnóstico de enfermedades genéticas prevalentes, incluyendo del SXF. Además, hay barreras económicas, políticas y sociales que enfrenta el campo neurogenético, principalmente en los países en desarrollo. ${ }^{2,3}$ Hasta la actualidad, el diagnóstico del SXF se basa principalmente en los hallazgos fenotípicos, con la posibilidad de realizar pruebas genéticas por recomendación del especialista. En muchos casos no se realizan debido a su alto costo, a que no están cubiertas por los seguros de salud y, en otros casos, a la poca disponibilidad de laboratorios certificados para realizar el análisis de ADN en muestras sanguíneas.

\section{Tratamiento}

No hay cura para el SXF, por lo que el tratamiento se limita al control de los síntomas asociados. Actualmente las líneas de investigación se centran en desarrollar tratamientos eficaces para los distintos problemas psiquiátricos y cognitivos que padecen los afectados (Tabla 3). En 2017, Gantois et al. investigaron la eficacia de metformina como modulador de la cascada de mGluR/mTORC1-ERK en modelos animales de SXF; reportaron mejoría en el comportamiento social y cognitivo, así como en las anormalidades morfológicas (disgenesia de la espinas dendríticas en el hipocampo) y electrofisiológicas (depresión a largo plazo).. ${ }^{28}$ Estos hallazgos motivaron el inicio de la investigación del tratamiento con metformina en la práctica clínica. El primer reporte demostró beneficio principalmente en comportamientos problemáticos como irritabilidad, agresividad y evasión social en pacientes adultos con SXF, además de beneficiar el control del apetito y el peso en quienes presentaban el fenotipo de Prader-Willi. ${ }^{29}$ Por esta razón, en la actualidad estudios controlados tanto en Estados Unidos como en Canadá buscan determinar la eficacia de metformina como tratamiento de este síndrome.

La sertralina es un medicamento de primera línea para el manejo de la depresión y la ansiedad. Fue estudiado por su potencial beneficio en el lenguaje, sin embargo, demostró mejores resultados en habilidades perceptuales motoras y visuales y participación social en SXF. ${ }^{21}$

La minociclina también se considera un tratamiento beneficioso en SXF. Se conoce que reduce los niveles de la matriz metalopeptidasa 9 (MMP-9), ${ }^{30}$ endopeptidasa dependiente de cinc encargada de regular la actividad sináptica crítica para el desarrollo y la plasticidad del sistema nervioso central. ${ }^{31}$ Su inhibición se produce por la unión con FMRP, proteína que se encuentra ausente en el SXF. Los problemas de regulación de MMP-9 se consideran parte de la fisiopatología no solo de los problemas de aprendizaje, sino también de las anomalías encontradas en el tejido conectivo. ${ }^{18}$

El acamprosato, un antagonista del receptor mGluR5, modificó el comportamiento ansioso y las pruebas locomotoras en el modelo animal de $\operatorname{SXF}^{32} y$ demostró mejoría en áreas de comportamiento social e hiperactividad en pacientes pediátricos con TEA y SXF. ${ }^{33}$ Debe considerarse un medicamento beneficioso para el manejo de los pacientes con SXF y problemas de adicción al alcohol. ${ }^{34}$

Los estudios de tratamiento con lovastatina en modelos animales de SXF postulan este medicamento como un tratamiento profiláctico para la epileptogénesis y sugieren que podría mejorar las funciones sensoriales y cognitivas. ${ }^{35}$ Los ensayos clínicos no controlados demostraron buena tolerancia en el tratamiento con pocos efectos adversos y reportaron beneficios tanto en el comportamiento como en las habilidades adaptativas ${ }^{36} \mathrm{~A}$ nivel molecular se demostró que los cambios en la fosforilación de la cinasa regulada por señal extracelular (ERK) se relacionan con la respuesta clínica a lovastatina. ${ }^{37}$

Existen otros medicamentos que pueden mejorar los sistemas neurobiológicos en SXF que no se consideran tratamientos específicos para el síndrome, sino que ayudan a controlar las características psiquiátricas más comunes. Estos incluyen los estimulantes (metilfenidato y anfetaminas) y atomoxetina, que 
Tabla 3. Medicamentos con eficacia en el tratamiento de síndrome $\mathbf{X}$ frágil

\begin{tabular}{|c|c|c|}
\hline Medicamento & Dosis máxima/día & Efectos adversos comunes \\
\hline Metformina & $\begin{array}{l}1000 \mathrm{mg}<50 \mathrm{~kg} \\
2000 \mathrm{mg}>50 \mathrm{~kg}\end{array}$ & Náusea, diarrea, cefalea, pérdida de peso \\
\hline Sertralina & $\begin{array}{c}2.5 \text { a } 5.0 \text { mg niños de } 2 \text { a } 6 \text { años } \\
10 \text { a } 100 \text { mg niños mayores de } 6 \text { años y adolescentes }\end{array}$ & Diarrea, pérdida de apetito, hiperhidrosis, temblor \\
\hline Minociclina & $\begin{array}{l}25 \mathrm{mg}<25 \mathrm{~kg} \\
50 \mathrm{mg} 25-45 \mathrm{~kg} \\
100 \mathrm{mg}>45 \mathrm{~kg}\end{array}$ & $\begin{array}{l}\text { Náusea, diarrea, cefalea, mareo, pérdida de apetito, } \\
\text { decoloración dental y de la cavidad oral, erupciones cutáneas }\end{array}$ \\
\hline Lovastatina & $40 \mathrm{mg}$ & $\begin{array}{l}\text { Debilidad, síntomas gastrointestinales, molestias musculares, } \\
\text { mareo, cefalea, irritabilidad }\end{array}$ \\
\hline Acamprosato & $\begin{array}{l}1332 \mathrm{mg}<50 \mathrm{~kg} \\
1998 \mathrm{mg}>50 \mathrm{~kg}\end{array}$ & $\begin{array}{l}\text { Irritabilidad, síntomas depresivos, aumento del comportamiento } \\
\text { repetitivo, síntomas gastrointestinales, incluyendo diarrea } \\
\text { y estreñimiento }\end{array}$ \\
\hline
\end{tabular}

pueden mejorar los síntomas del trastorno de atención e hiperactividad, por lo general, en niños mayores de cinco años; también se pueden usar los agonistas alfa adrenérgicos (guanfacina o clonidina) antes de los cinco años para calmar la hiperactividad. La clonidina es especialmente eficaz para mejorar los trastornos del sueño, de no tener una buena respuesta al tratamiento con melatonina. Para el manejo de la agresividad o los desórdenes del estado de ánimo, los antipsicóticos (risperidona o aripiprazol) son adecuados, pero pueden causar aumento de peso.

\section{Conclusión}

Los individuos afectados con el SXF presentan discapacidad intelectual, TEA y TDAH. Aunque existen muchos medicamentos para el manejo de las comorbilidades comunes, no hay tratamientos específicos. El objetivo del tratamiento temprano es mejorar la discapacidad intelectual, las dificultades de la comunicación y la interacción social característicos del SXF. Además, a pesar de la recomendación de realizar pruebas genéticas en niños con discapacidad intelectual o retraso global del desarrollo, estas no se realizan en muchos de los países latinoamericanos. Es de mayor importancia implementar el análisis SXF en todos los países hispanos.

\section{Financiamiento}

Esta investigación fue apoyada por el Instituto Nacional de Desórdenes Neurológicos y Accidentes Cerebrovasculares, con el financiamiento 1NS107131; por el Instituto Nacional de Salud Mental, subvención MH094681; el Instituto Nacional de Salud Infantil y
Desarrollo Humano, financiamientos R01 HD036071 y U54 HD079125, para el Centro de Investigación de Discapacidad Intelectual y del Desarrollo del Instituto Médico de Investigación del Neurodesarrollo, Universidad de California en Davis y el Hospital Infantil Shriners. El contenido de este trabajo es responsabilidad exclusiva de los autores y no representa necesariamente las opiniones oficiales de las instituciones financiadoras. Además, dichas agencias no respaldan la compra de ningún producto o servicio comercial mencionado en la publicación.

\section{Conflictos de interés}

Randi J. Hagerman ha recibido fondos de Roche, Novartis, Neuren, Marinus y Alcobra para llevar a cabo estudios de tratamiento en pacientes con SXF. También ha consultado con Fulcrum y Zynerba sobre estudios de tratamiento en individuos con SXF. Los demás autores declaran no tener conflictos de interés.

\section{Referencias}

1. Hagerman PJ. The fragile X prevalence paradox. J Med Genet. 2008; 45:498-499.

2. Fernández-Carvajal I, Walichiewicz P, Xiaosen X, Pan R, Hagerman PJ, Tassone F. Screening for expanded alleles of the FMR1 gene in blood spots from newborn males in a Spanish population. J Mol Diagn. 2009; 11:324-329.

3. Saldarriaga W, Forero-Forero JV, González-Teshima LY, Fandiño-Losada A, Isaza C, Tovar-Cuevas JR, et al. Genetic cluster of fragile X syndrome in a Colombian district. J Hum Genet. 2018;63:509-516.

4. Danesi C, Achuta VS, Corcoran P, Peteri UK, Turconi G, Matsui N, et al Increased calcium influx through L-type calcium channels in human and mouse neural progenitors lacking fragile $X$ mental retardation protein. Stem Cell Reports. 2018;11:1449-1461.

5. Gatto CL, Pereira D, Broadie K. GABAergic circuit dysfunction in the Drosophila fragile $X$ syndrome model. Neurobiol Dis. 2014;65:142-159.

6. Higashimori H, Morel L, Huth J Lindemann L, Dulla C, Taylor A, et al. Astroglial FMRP-dependent translational down-regulation of mGluR5 underlies glutamate transporter GLT1 dysregulation in the fragile X mouse. Hum Mol Genet. 2013;22:2041-2054. 
7. Wang H, Ku L, Osterhout DJ, Li W, Ahmadian A, Liang Z, et al. Developmentally-programmed FMRP expression in oligodendrocytes: a potential role of FMRP in regulating translation in oligodendroglia progenitors. Hum Mol Genet. 2004;13:79-89.

8. Pilaz LJ, Lennox AL, Rouanet JP, Silver DL. Dynamic mRNA transport and local translation in radial glial progenitors of the developing brain. Curr Biol. 2016;26:3383-3392.

9. Brown MR, Kronengold J, Gazula VR, Chen Y, Strumbos JG, Sigworth FJ, et al. Fragile $X$ mental retardation protein controls gating of the sodium-activated potassium channel Slack. Nat Neurosci. 2010;13:819-821.

10. Deng PY, Rotman Z, Blundon JA, Cho Y, Cui J, Cavalli V et al. FMRP regulates neurotransmitter release and synaptic information transmission by modulating action potential duration via BK channels. Neuron. 2013;77:696-711.

11. Dockendorff TC, Labrador $M$. The fragile $X$ protein and genome function. Mol Neurobiol. 2018:56:711-721.

12. Westmark CJ. Fragile $X$ and APP: a decade in review, a vision for the future. Mol Neurobiol. 2019;56:3904-3921.

13. Hagerman RJ, Berry-Kravis E, Hazlett HC, Bailey DB Jr, Moine $H$, Kooy RF, et al. Fragile X syndrome. Nat Rev Dis Primers. 2017:3:17065.

14. Bailey DB Jr, Raspa M, Olmsted M, Holiday DB. Co-occurring conditions associated with FMR1 gene variations: findings from a national parent survey. Am J Med Genet A. 2008;146A:2060-2069.

15. Hagerman RJ, Hagerman $P$. Fragile X-associated tremor/ataxia syndrome-features, mechanisms and management. Nat Rev Neurol. 2016;12:403-412.

16. Salcedo-Arellano MJ, Hagerman RJ, Martínez-Cerdeño V. Síndrome de temblor y ataxia asociado al X frágil (FXTAS): presentación clínica, patología, y tratamiento. Rev Neurolo. 2019;68:199-206.

17. Pretto D, Yrigollen CM, Tang HT, Williamson J, Espinal G1, Iwahashi CK et al. Clinical and molecular implications of mosaicism in FMR1 full mutations. Front Genet. 2014;5:318.

18. Ramírez-Cheyne JA, Duque GA, Ayala-Zapata S, Saldarriaga-Gil W, Hagerman $P$, Hagerman $R$, et al. Fragile $X$ syndrome and connective tissue dysregulation. Clin Genet. 2018;95:262-267.

19. Thurman AJ, McDuffie A, Hagerman R, Abbeduto L. Psychiatric symptoms in boys with fragile $\mathrm{X}$ syndrome: a comparison with nonsyndromic autism spectrum disorder. Res Dev Disabil. 2014;35:1072-1086.

20. Berry-Kravis EM, Hessl D, Rathmell B, Zarevics P, Cherubini M, Walton-Bowen K, et al. Effects of STX209 (arbaclofen) on neurobehavioral function in children and adults with fragile $X$ syndrome: a randomized, controlled, phase 2 trial. Sci Transl Med. 2012:4:152ra127.

21. Greiss-Hess L, Fitzpatrick SE, Nguyen DV, Chen Y, Gaul KN Schneider A, et al. A randomized, double-blind, placebo-controlled tria of low-dose sertraline in young children with fragile $X$ syndrome. J Dev Behav Pediatr. 2016;37:619-628

22. Bailey DB Jr, Raspa M, Bishop E, Holiday D. No change in the age of diagnosis for fragile $\mathrm{x}$ syndrome: findings from a national parent survey. Pediatrics. 2009:124:527-533.

23. Tassone F, Pan R, Amiri K, Taylor AK, Hagerman PJ. A rapid polymerase chain reaction-based screening method for identification of all expanded alleles of the fragile X (FMR1) gene in newborn and high-risk populations. J Mol Diagn. 2008;10:43-49.
24. Tejada MI. Prevention of fragile $X$ syndrome by prenatal genetic diagnosis: advantages and controversial aspects. Rev Neurol. 2001;33: S14-S19.

25. Riley $C$, Wheeler $A$. Assessing the fragile $X$ syndrome newborn screening landscape. Pediatrics. 2017;139:S207-S215.

26. Moeschler JB, Shevell M, Committee on Genetics. Comprehensive evaluation of the child with intellectual disability or global developmental delays. Pediatrics. 2014;134:e903-918.

27. Lubala TK, Lumaka A, Kanteng G, Mutesa L, Mukuku O, Wembonyama S, et al. Fragile X checklists: a meta-analysis and development of a simplified universal clinical checklist. Mol Genet Genomic Med. 2018; 6:526-532.

28. Gantois I, Khoutorsky A, Popic J, Aguilar-Valles A, Freemantle E, Cao R, et al. Metformin ameliorates core deficits in a mouse model of fragile $X$ syndrome. Nat Med. 2017;23:674-677.

29. Dy ABC, Tassone F, Eldeeb M, Salcedo-Arellano MJ, Tartaglia N, Hagerman R. Metformin as targeted treatment in fragile X syndrome. Clin Genet. 2018:93:216-222

30. Dziembowska M, Pretto DI, Janusz A, Kaczmarek L, Leigh MJ, Gabriel N, et al. High MMP-9 activity levels in fragile $X$ syndrome are lowered by minocycline. Am J Med Genet A. 2013;161A:1897-1903.

31. Ethell IM, Ethell DW. Matrix metalloproteinases in brain development and remodeling: synaptic functions and targets. J Neurosci Res. 2007:85: 2813-2823

32. Schaefer TL, Davenport MH, Grainger LM, Robinson CK, Earnheart AT, Stegman MS, et al. Acamprosate in a mouse model of fragile $X$ syndrome: modulation of spontaneous cortical activity, ERK1/2 activation, locomotor behavior, and anxiety. J Neurodev Disord. 2017;9:6.

33. Erickson CA, Wink LK, Early MC, Stiegelmeyer E, Mathieu-Frasier L, Patrick V, et al. Brief report: pilot single-blind placebo lead-in study of acamprosate in youth with autistic disorder. J Autism Dev Disord. 2014;44:981-987.

34. Salcedo-Arellano MJ, Lozano R, Tassone F, Hagerman RJ, Saldarriaga W. Alcohol use dependence in fragile X syndrome. Intractable Rare Dis Res. 2016;5:207-213

35. Osterweil EK, Chuang SC, Chubykin AA, Sidorov M, Bianchi R, Wong RK, et al. Lovastatin corrects excess protein synthesis and prevents epileptogenesis in a mouse model of fragile X syndrome. Neuron. 2013; $77: 243-250$

36. Caku A, Pellerin D, Bouvier P, Riou E, Corbin F. Effect of lovastatin on behavior in children and adults with fragile $X$ syndrome: an open-label study. Am J Med Genet A. 2014;164:2834-2842.

37. Pellerin D, Caku A, Fradet M, Bouvier P, Dubé J, Corbin F. Lovastatin corrects ERK pathway hyperactivation in fragile $X$ syndrome: potential of platelet's signaling cascades as new outcome measures in clinical trials. Biomarkers. 2016;21:497-508.

38. Leigh MJ, Nguyen DV, Mu Y, Winarni TI, Schneider A, Chechi T, et al. A randomized double-blind, placebo-controlled trial of minocycline in children and adolescents with fragile $\mathrm{x}$ syndrome. J Dev Behav Pediatr. 2013;34:147-155 